\title{
Vitrification of Human Oocytes and its Contribution to In Vitro Fertilization Programs
}

Carlos G. Almodinn ${ }^{1,2}$, Alvaro Ceschin ${ }^{3}$, Raul E. Nakano4, Moacir R.M. Radaelli1,5, Paula M. Almodin ${ }^{1}$, Carla G. Silva ${ }^{1}$, Lucilene K. Nishikawa ${ }^{3}$, Litsuko S. Fujihara ${ }^{4}$, Vânia C. Minguetti-Câmara ${ }^{1,6}$

${ }^{1}$ Materbaby - Reprodução Humana e Genética - Maringá/PR, Brazil

${ }^{2}$ Departamento de Obstetrícia, Universidade Federal de São Paulo - UNIFESP - São Paulo/SP, Brazil

${ }^{3}$ Feliccità - Instituto de Fertilidade - Curitiba/PR, Brazil

${ }^{4}$ Ferticlin - Clínica de Reprodução Humana - São Paulo, Brazil

${ }^{5}$ Departamento de Cirurgia, Santa Casa de São Paulo Escola de Ciências Médicas - São Paulo/SP, Brazil

${ }^{6}$ Departamento de Análise Farmacêutica, Universidade Estadual de Maringá - UEM - Maringá/PR, Brazil

\begin{abstract}
Objeticve: To study the cumulative pregnancy outcome, particularly in terms of live births, with the consecutive transfer of embryos from fresh and vitrified/warmed oocytes to infertile patients in a routine infertility program. Methods: Patients were initially submitted to in vitro fertilization embryo transfer with fresh embryos, while surplus oocytes were vitrified with the Vitri-Ingá method. Patients who did not succeed to carry their gestation to term underwent a new cycle with embryos from their own warmed oocytes. Some of the patients participating in the first warming cycle, who still possessed surplus oocytes, underwent a second warming cycle. Clinical and pregnancy outcomes obtained with fresh and warming cycles were compared using the chi-square test at a level of significance of $5 \%$.
\end{abstract}

Results: Of the 211 participating patients, 97 (46\%) got pregnant with fresh embryo transfer, and 69 (32.7\%) carried their pregnancies to term. Of the patients participating in the first and second warming cycles, 32/100 (32\%) and $6 / 20(30.0 \%)$ resulted in live births, respectively. Thus, of the 211 participating patients, 107 carried their pregnancies to term, representing a cumulative live birth rate of $50.7 \%$. No statistically significant differences between the use fresh and vitrified oocytes were found for any of the variables studied.

Conclusions: Oocyte vitrification offered the possibility of gestation in more than one attempt after just one controlled hyperstimulation. Apart from alleviating the financial burden on patients, vitrification of oocytes may result in a feasible solution for the problems generated by abandoned frozen embryos.

Keywords: Human Oocyte, Vitrification, Pregnancy Rates

\section{INTRODUCTION}

The advent of ovulation induction in assisted reproduction fertilization cycles has resulted in the production of supernumerary oocytes and embryos which needed to be cryopreserved and stored to be used at a later date. Since the first report by Trounson \& Mohr (1983) on the successful use of frozen/thawed embryos, the cryopreservation of embryos has become routine in human reproduction centers worldwide. The cryopreservation of embryos in in vitro fertilization (IVF) programs has resulted in increased pregnancy rates while, at the same time, avoided the undesirable consequences of transferring large numbers of embryos at one time (Wang et al., 2008; Zhou et al., 2009; Wong et al., 2014).

A possible drawback of the embryo cryopreservation strategy, however, is the accumulation of cryostored embryos, which are sometimes abandoned in human reproduction centers and may have an uncertain destiny. This is particularly the case when one of the spouses die or cou- ples divorce either litigiously or consensually (Robertson, 1989; Tucker et al., 1995; Craft \& Forman, 1996). Thus, the decision of what to do with such embryos can raise serious legal, moral and religious concerns.

Recently, the cryopreservation of oocytes has emerged as a possible solution to the problem of storing unwanted embryos (Borini et al., 2008; Cobo et al., 2008; Rienzi et al., 2010). The cryopreservation of oocytes has been generally indicated in cases of oncologic patients submitted to chemo/radiotherapy treatments, and also for women without medical problems who desire to extend their reproductive lifespan, regardless of having a partner. In IVF programs, more specifically, the cryopreservation of oocytes has the potential to benefit patients suffering from different conditions such as poor responders and ovarian hyperstimulation syndrome, or when semen collection from the husband on the day that insemination is to be performed fails (Cil \& Seli, 2013).

Vitrification, a rapidly developing technology, which is increasingly being used for the cryopreservation of gametes, embryos (particularly blastocysts) and germinative tissue, has been demonstrated to be a reliable strategy for the cryopreservation of oocytes (Cobo et al., 2008; Cobo et al., 2010; Almodin et al., 2010). The latest systematic review and meta-analysis comparing vitrified with fresh oocytes demonstrated no statistically significant differences in fertilization, cleavage and clinical pregnancy rates, although ongoing pregnancy rate was reduced in the vitrified group (Potdar et al., 2014). Moreover, according to the Practice Committees of the American Society for Reproductive Medicine and the Society for Assisted Reproductive Technology, there is good evidence that fertilization and pregnancy rates obtained with vitrified/warmed oocytes are similar to fresh oocytes when used as part of IVF by intracytoplasmic sperm injection (ICSI) for young women (Pfeifer et al., 2013).

As vitrification methods become more reliable and widespread, oocyte vitrification emerges as an important alternative to the more traditional cryopreservation methods and strategies (Kuwayama et al., 2005; Cobo et al., 2008; Rienzi et al., 2010; Siano et al., 2013). Oocyte vitrification has the potential to substantially reduce the number of abandoned embryos in human reproduction practices, offer the possibility of pregnancy in more than one attempt with just one ovulation induction, while unused/ unwanted oocytes could help foster oocyte banks (Cobo et al., 2010; Ubaldi et al., 2010). However, in order to demonstrate the potential benefits that the vitrification of oocytes may bring to patients and to global IVF success rates, further clinical studies are still necessary to establish the feasibility of the strategy.

Therefore, the objective of this prospective cohort clinical study was to assess the cumulative outcome, particu- 
larly in terms of live births, with the consecutive transference of embryos from fresh and vitrified/thawed oocytes to infertile patients in a routine infertility program.

\section{MATERIALS AND METHODS Ethical Approval}

This multicenter prospective cohort clinical study was analyzed and approved by the institutional review board at Materbaby - Reprodução Humana e Genética (protocol no 17-2011), in compliance with the Brazilian Ethical Norms on the Use of Assisted Reproduction Techniques (CFM no $1.957 / 2010,15$ th December). Patients were thoroughly informed on the procedures and objectives of the study, and their participation was granted only after the signature of a free informed consent.

\section{Study design and patient inclusion}

The procedures were performed at the following human reproduction centers: Ferticlin - Centro de Fertilidade Humana (São Paulo, Brazil), Feliccitá - Instituto de Fertilidade (Curitiba, Brazil) and Materbaby - Reprodução Humana e Genética (Maringá, Brazil).

Only those patients with at least 10 oocytes in metaphase II (MII) were included in the study. All the patients underwent only one fresh embryo transfer attempt, and those patients that did not carry their gestation to term had the option to use their surplus vitrified oocytes in new embryo transfer cycles. All patients were consecutively treated between January 2011 and December 2012.

\section{Oocyte pickup, IVF, embryo culture and transfer}

Controlled ovarian hyperstimulation was performed with the long protocol using pituitary suppression with gonadotropin-releasing hormone $(\mathrm{GnRH})$-agonist (Lupron ${ }^{\circledR}$ - Abbot Laboratories, São Paulo, Brazil) in mid-luteal phase of the preceding cycle. Recombinant follicle-stimulating hormone ( $r-F S H)$ (Gonal-F® - Merck-Serono, São Paulo, Brazil) associated with human menopausal gonadotropin (hMG) (Menopur $\AA$ - Ferring Pharmaceuticals, São Paulo, Brazil) was administered on the second day of the menstrual cycle and kept until follicular dominance. Follicle growth was monitored by ultrasound, and either urinary human chorionic gonadotropin (u-hCG) 10,000 IU (Choriomom $\AA$ - Meizler, São Paulo, Brazil), or recombinant human chorionic gonadotropin (r-hCG) $250 \mu \mathrm{g}$ (Ovidrel ${ }^{\circledR}$ - Merck-Serono, São Paulo, Brazil) was administered when at least two leading follicles $\geq 18 \mathrm{~mm}$ in diameter were observed. Oocyte retrieval was performed 36 hours after hCG administration with the assistance of transvaginal ultrasound. After retrieval, oocytes were transferred to an incubator with $6 \% \mathrm{CO} 2$ at $37^{\circ} \mathrm{C}$, and one to two hours later placed in hyaluronidase (IngaMed, Maringá, Brazil) for the removal of cumulus cells. A total of 6 oocytes in MII were separated to be inseminated by ICSI using polyvinylpyrrolidone (PVP - IngaMed, Maringá, Brazil), while the remaining oocytes in MII were vitrified.

Embryo culture was conducted in a $50 \mu \mathrm{l}$ microdrop of culture medium (GV-Blast $\AA$ - IngaMed, Maringá, Brazil) under oil. Fertilization was assessed 18-20 h after ICSI, and embryos were kept in culture until day three post-fertilization as previously reported (Almodin et al., 2010). Good quality, Grade I embryos were defined as those with 6-8 cells, similarly-shaped blastomeres and no fragmentation, while Grade II embryos as those with 6-8 cells and similarly-shaped blastomeres with $\leq 20 \%$ fragmentation (Alikhani et al., 1999). Before transfer, an assessment of the total number of embryos was performed, and embryos not selected for transfer were vitrified and stored into a nitrogen tank.

Ultrasound guided embryo transfer was performed. Luteal support was conducted with injectable progesterone
(West-Ward Pharmaceuticals, NJ, USA) $50 \mathrm{mg} / \mathrm{ml}$ IM once daily, or intravaginal micronized progesterone (Utroges$\tan \AA$ - Farmoquimica, São Paulo, Brazil) 800 mg daily, until the pregnancy test. Gestation was confirmed by $\beta$-hCG level on day 14 after embryo transfer, in which case the same protocol was kept until week 10 of gestation.

Implantation rate was defined as the number of gestational sacs per transferred embryo. Chemical pregnancy was determined in those cases when $\beta$-hCG above 25 mUI/ $\mathrm{ml}$ was detected without, however, the visualization of the gestational sac, and clinical pregnancy when heartbeat was detected. Miscarriage was defined as the interruption of gestation after the visualization of a gestational sac, with or without the presence of heartbeat.

\section{Oocyte vitrification and warming}

The Vitri-Ingá method employed for oocyte vitrification was that described previously by Almodin et al., 2010. All materials required for vitrification/warming were obtained from Ingamed (Maringá, Brazil).

Briefly, the vitrification process started by transferring the oocytes to the equilibrium solution VI-1, where they stayed for 9-15 min. After that, each oocyte was individually transferred to three consecutive $20 \mu$ drops of vitrification solution VI-2, and then to the vitrification strip. A maximum of 2 oocytes were deposited on each vitrification strip with minimum vitrification solution, and then immediately immersed into liquid nitrogen. When more than 1 $\mu \mathrm{l}$ of the solution was deposited with the oocytes on the strip, excess liquid was aspirated before vitrification. The whole vitrification process, until the vitrification strip was immersed into liquid nitrogen, took no more than 50-70 s. For the safe storage of vitrified oocytes, the vitrification strips were inserted into sterile plastic straws, which were heat sealed.

Oocyte warming was conducted as follow: immediately after the vitrification strip was removed from the sealed plastic straw, it was immersed into of the warming solution DV-I, containing $1 \mathrm{M}$ of sucrose at $37^{\circ} \mathrm{C}$ for $1 \mathrm{~min}$. The oocytes were then transferred to the diluting solution DV-II, with $0.5 \mathrm{M}$ of sucrose for $3 \mathrm{~min}$ at room temperature, and rinsed twice in the buffer solution DV-III, 5 min each.

After warming had been completed, oocyte survival was assessed according to the morphological criteria: re-expansion, with intact cell membrane, normal ooplasm and zona pellucida, as well as perivitelline space of normal size. ICSI was performed on selected oocytes $2-3 \mathrm{~h}$ after warming.

Oocyte warming was conducted as follow: immediately after the vitrification strip was removed from the sealed plastic straw, it was immersed into of the warming solution DV-I, containing $1 \mathrm{M}$ of sucrose at $37^{\circ} \mathrm{C}$ for $1 \mathrm{~min}$. The oocytes were then transferred to the diluting solution DV-II, with $0.5 \mathrm{M}$ of sucrose for $3 \mathrm{~min}$ at room temperature, and rinsed twice in the buffer solution DV-III, 5 min each.

After warming was completed, oocyte survival was assessed according to the morphological criteria: re-expansion, with intact cell membrane, normal ooplasm and zona pellucida, as well as perivitelline space of normal size. ICSI was performed on selected oocytes $2-3 \mathrm{~h}$ after warming.

\section{Vitrified/Warmed Oocyte Cycles (VWOC)}

Patients that did not succeed to get pregnant or did not carry their gestation to term, and were willing to undergo a new attempt, were prepared for new cycles using their vitrified/warmed oocytes. Oral contraceptive (Microdiol ${ }^{\circ}$ Organon, São Paulo, Brazil) was initiated on day 5 of the menstrual cycle. On the day 20 of the cycle, an ampoule

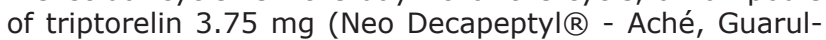
hos, Brazil) was administered, after which patients waited for their menstrual bleeding. Between day 2 and 4 of the 
menses, patients were submitted to transvaginal ultrasound examination and a serum oestradiol blood test was performed. When no ovarian cyst was observed with the ultrasound and oestradiol levels were less than $40 \mathrm{pg} / \mathrm{ml}$, oral oestradiol valerate (Primogyna, Schering, São Paulo, Brazil) was initiated $2 \mathrm{mg} /$ day for 6 days, followed by 4 $\mathrm{mg} /$ day for 3 days, and $6 \mathrm{mg} /$ day for 3 days, after which the patient was submitted to transvaginal ultrasound to assess the endometrium. When the endometrium was $\geq 7$ $\mathrm{mm}$ thick, the administration of injectable progesterone $100 \mathrm{mg} / \mathrm{IM}$ (West-Ward Pharmaceuticals), or intravaginal micronized progesterone (Utrogestan $®$ ) $800 \mathrm{mg} /$ day was initiated. Oocytes were warmed on the day progesterone was initiated and fertilized with ICSI. After embryo development and quality assessment, selected embryos were transferred on day 3 after progesterone initiation.

\section{Outcome Measures and Statistical Analysis}

Patients' baseline characteristics, embryological data, and pregnancy rates were analyzed on a per cycle basis. The cumulative success rates wereanalyzed on a perpatientbasis. Life birth cumulative rate was determined for fresh oocytes and vitrified oocytes of the same ovarian stimulation cycle.

Statistical significance was estimated using chi-square test at a level of significance of $0.05 \%(a=5 \%)$. Descriptive levels $(P)$ under this value were considered significant.

\section{RESULTS}

Figure 1 displays the participating patients and their progress over the experimental period.

\section{Oocyte vitrification/warming}

A total of 211 patients, aged between 27 and 41 years $(33.0 \pm 3.6)$, were included in the study. Table 1 displays the clinical outcomes of fresh and warming cycles. In comparison to fresh cycles, vitrified/warmed cycles presented no statistically significant differences concerning patients' age $(P=0.8707)$, fertilization rates $(P=0.1546)$, embryo quality $(P=0.6474)$, the number of transferred embryos $(P=0,0911)$ or implantation rates $(P=0.7314)$.

A total of 3260 oocytes were retrieved, of which 2650 were in metaphase II, while the other 610 oocytes were either immature or atretic, and were discarded. Insemination by ICSI was conducted in 1045 oocytes, while 1488 were vitrified for future attempts at pregnancy by the patients. Forty-four patients donated 117 surplus oocytes to the oocyte bank to be used by other patients, which were also vitrified. Eighteen hours after ICSI, 882 oocytes $(84.4 \%)$ were successfully fertilized. The assess- ment conducted on the day three of embryo development, 457 (52\%) embryos were deemed Grade I or II. A total of 516 fresh embryos were transferred $(2.4 \pm 0.6)$ to the 211 participating patients.

Of the 1488 vitrified oocytes, 511 were warmed in the two vitrified/warmed cycles (VWCs), among which $458(89.6 \%)$ survived and were inseminated by ICSI, which resulted in a fertilization rate of $81.4 \%(373 / 458)$. Grade I and II embryos on day three represented $50.4 \%$ $(188 / 373)$, and a total of 272 embryos $(2.3 \pm 0.9)$ were transferred in 120 VWCs.

\section{Pregnancy Rates}

Table 2 displays the pregnancy rate results for fresh embryo cycle and the first and second VWCs. No statistically significant differences were found between fresh and VWCS concerning pregnancy rate $(P=0.3654)$, clinical pregnancy $(P=0.4744)$, chemical pregnancy $(P=$ $0.6624)$, pregnancy to term $(P=0.8466)$ or the number of abortions $(P=0.3618)$.

Of the 211 patients included in the study, 97 (46\%) presented positive $\beta$ hCG ( $\geq 25 \mathrm{mUI} / \mathrm{ml})$ after fresh embryo transfer, among whom $84(39.3 \%)$ presented clinical pregnancy, and 69 carried their gestation to term. Of the 114 patients with negative $\beta$-hCG $(<25 \mathrm{mUI} / \mathrm{ml}), 80$ decided for a new attempt (first VWC). Likewise, 20 of the 28 patients who got pregnant in their first attempt with fresh embryos but did not succeed in having a baby also decided for their first VWC, totaling 100 patients.

Of the 100 patients in the first VWC, $40(40 \%)$ presented positive $\beta$-hCG, 36 (36\%) were clinically pregnant, and $32(32 \%)$ patients carried their gestation to term. Of the 68 patients participating in the first VWC, who did not succeed in having a baby, 51 still had surplus vitrified oocytes for a second attempt. Of the 51 patients, 20 opted for the second VWC, from whom $9(45 \%)$ was $\beta$-hCG positive, 7 (35\%) demonstrated clinical pregnancy, and 6 (30\%) carried their pregnancy to term.

Thus, of the 211 patients included in the study, a cumulative pregnancy to term rate of $50.7 \%(107 / 211)$ was obtained after the fresh and the first and second VWCs. Of the 104 patients yet to have babies, 32 did not have any remaining oocytes for new attempts. However, 72 patients (791 oocytes) can still undergo their first or a new VWC.

After the second VWC, 11 patients who had no intention to perform a new IVF attempt, either because they had already had babies or decided not to proceed with the treatment, donated 67 oocytes to the oocyte bank. All the donated vitrified cryo-banked oocytes were quarantined

Figure 1: Pregnancy rates after fresh and the 1st and 2nd vitrified/warmed cycle (VWC)

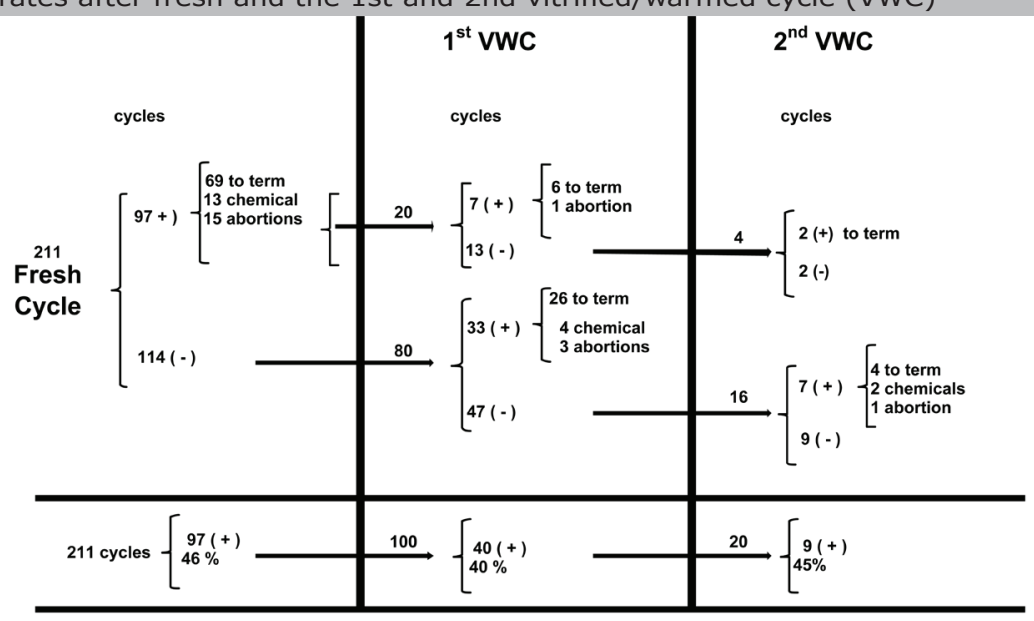

Pregnancies to term / Cumulative rate $($ fresh + vitrified/warmed oocytes $)=107 / 211(50.7 \%)$ 
Table 1: Clinical outcomes of fresh and vitrified/warmed cycles

\begin{tabular}{|c|c|c|c|}
\hline & Fresh cycle & $1^{\text {st }}+2^{\text {nd }}$ VWC & P-value \\
\hline Cycles & 211 & 120 & \\
\hline age (mean \pm SD) & $33.0 \pm 3.6$ & $33.1 \pm 3.2$ & 0.8707 \\
\hline Total collected oocytes & 3260 & - & \\
\hline Total mature oocytes & 2650 & - & \\
\hline Mature oocytes (mean \pm SD) & $12.6 \pm 4.3$ & - & \\
\hline Vitrified oocytes & 1488 & - & \\
\hline Thawed oocytes & - & 511 & \\
\hline Surviving oocytes (survival rate \%) & - & $458(89.6)$ & \\
\hline Injected oocytes & 1045 & 458 & \\
\hline Fertilized oocytes (fertilization rate \%) & $882 / 1045(84.4)$ & $373 / 458(81.4)$ & 0.1546 \\
\hline Embryos type I or II (quality rate \%) & $457 / 882(51.8)$ & $188 / 373(50.4)$ & 0.6474 \\
\hline Transferred embryos & 516 & 272 & \\
\hline Transferred embryos (mean \pm SD) & $2.4 \pm 0.6$ & $2.3 \pm 0.9$ & 0.0911 \\
\hline $\mathbf{N}^{\circ}$ gestational sacs (Implantation rate \%) & $102 / 516(19.8)$ & $51 / 272(18.8)$ & 0.7314 \\
\hline
\end{tabular}

VWC: Vitrified/Warmed cycle

Table 2: Pregnancy rates after fresh and vitrified/warmed cycles

Table 2: Pregnancy rates after fresh and vitrified/warmed cycles
\begin{tabular}{|l|c|c|c|}
\hline & Fresh transfer & $\mathbf{1}^{\text {st }}+\mathbf{2}^{\text {nd }}$ VWC & P-value \\
\hline & 211 & 120 & \\
\hline Pregnancies per transfer (\%) & $97 / 211(46.0)$ & $49 / 120(40.8)$ & 0.3654 \\
\hline Clinical pregnancies per transfer (\%) & $84 / 211(39.8)$ & $43 / 120(35.8)$ & 0.4744 \\
\hline Chemical pregnancies per transfer (\%) & $13 / 211(6.2)$ & $6 / 120(5.0)$ & 0.6624 \\
\hline Pregnancies to term per transfer (\%) & $69 / 211(32.7)$ & $38 / 120(31.7)$ & 0.8466 \\
\hline Abortions (\%) & $15 / 84(17.9)$ & $5 / 43(11.6)$ & 0.3618 \\
\hline
\end{tabular}

VWC: Vitrified/Warmed cycle

for a minimum of 6 months and were assigned only after the seronegativity of the donor was verified. Other 13 patients, who did not wish to donate their oocytes, had their 119 remaining oocytes discarded.

\section{DISCUSSION}

This multicenter prospective cohort clinical study demonstrated that the vitrification of supernumerary oocytes is a feasible strategy that can increase live birth rates without the need for new ovulation inductions, clinically and financially benefitting patients.

The vitrification method used in the present study, the Vitri-Ingá system, has been routinely used in the services involved in the present study. This system permits vitrification to be conducted with the use of minimum volume of cryoprotectant, increasing cooling and warming rates and, consequently, decreasing cell damage (Almodin et al., 2010). The Vitri-Ingá method seemed to be effective and safe in the cryopreservation of oocytes in the sample studied. The results of this study demonstrated that the oocyte vitrification method used did not have a negative effect on the fertilization rates and embryo quality. The similar fertilization rates observed with both fresh and frozen/thawed oocytes, suggested that not only the morphological but also the functional integrity of oocytes were well preserved with vitrification. Thus, fresh embryo quality on day 3 was not significantly different from that of similar embryos from vitrified oocytes. Nonetheless, future research is needed to consolidate the method's safety and give information about health of children born from vitrified/thawed oocytes.

It is well known that, embryo quality as well as embryo development synchronism with endometrial receptivity are important factors that can affect pregnancy rates in IVF cycles (Coutifaris et al., 2004; Loutridis et al., 2008). Recent studies in animals and humans suggest that ovarian hyperstimulation, an important part of the IVF procedures, can lead to several alterations in the endometrium, such as epithelium depth, the number and length of microvilli, the mitotic activity on the epithelial interface with stromal cells, and decreased number of specific integrins associated with the implantation window (Chen et al., 2007; Al-Ghamdi et al., 2008; Weinerman \& Mainigi, 2014). Moreover, during fresh IVF cycles, multiple formations of corpora lutea secrete high levels of hormones and other factors that may affect the endometrium and, consequently, embryo implantation in the uterus (Weinerman \& Mainigi, 2014).

Endometrial receptivity may be more affected in those women with an exaggerated response to ovarian stimulation. This is probably due to the fact that these patients have increased levels of estrogen and progesterone during and after the superovulation. Clinical studies indicate that high levels of estrogen are correlated with early progesterone peaks, even before hCG is administered to trigger the ovulation (Kyrou et al., 2009; Bosch et al., 2010; Kolibianakis et al., 2012; Al-Azemi et al., 2012). High levels of progesterone have been associated with the early secretion stage in human endometrium and a drop in pregnancy rates (Weineman \& Mainigi, 2014). Comparative studies using endometrial biopsies obtained during natural and stimulated cycles demonstrated that changes in the endometrial gene expression, coinciding with 2 to 4 days advancement of the endometrial implantation window, occurred in cases of ovarian hyperstimulation (Zhang et 
al., 2008; Ruiz-Alonso et al., 2013). Other risks associated with the transfer of fresh embryos after ovarian hyperstimulation include pre-eclampsia, low birth weight, prematurity, bleeding at delivery, placental abruption and perinatal death (Shapiro et al., 2014).

As a result, some recent studies have suggested that embryo transfer should be performed only in post-cryopreservation/thawing cycles, which would permit the endometrium to be better prepared in natural cycles (Barnhart, 2014; Shapiro et al., 2014; Weinerman \& Mainigi, 2014). In an attempt to obtain the best embryo/endometrium synchronism, Zhou et al. (2009) conducted a fresh embryo transfer cancellation program with later transfer of frozen/thawed embryos, which resulted in improved pregnancy rates.

Natural cycles, or cycles with hormone replacement, similar to natural cycles, used in cases of cryopreservation/ warming of embryos and oocytes could, therefore, result in improved endometrial receptivity (Chang et al., 2011; Levron et al., 2014). It is believed that the transfer of frozen/ thawed embryos is associated with less risk of implantation failure when compared with fresh embryo transfer (Shapiro et al., 2014).

The vitrification of oocytes may represent an advantage over the cryopreservation of embryos. Routinely, toprate embryos are transferred fresh and only second best embryos are cryopreserved. Their use in frozen cycles may compromise results, despite the still theoretical advantage obtained with improved endometrial receptivity of a natural cycle (Shapiro et al., 2014; Wang et al., 2014). Vitrified oocytes, on the other hand, have been shown to produce embryos with quality similar to fresh oocytes (Potdar et al., 2014). The choice for the best embryos derived from vitrified oocytes may bring an important contribution to the delayed implantation strategy. Further studies should be conducted to ascertain this assumption.

Apart from the biological aspects, the vitrification of oocytes may bring some other additional noteworthy benefits to patients. It has been previously reported that the use of cryopreserved embryos can reduce the overall costs of IVF treatment. Veleva et al. (2009) compared IVF clinical results during the 1995-1999 period, when the transfer of cryopreserved/thawed embryos was seldom employed $(4.2 \%)$, with the 2000-2004 period, when the number of transfers had sharply grown $(46.2 \%)$. The cost/benefit ratio per birth calculated based on the costs of the treatment and medications, suggested that the transfer of just one selected top-quality embryo associated to cryopreservation could improve results and decrease IVF costs. Following the same rationale, the vitrification of surplus oocytes may also lead to reductions in the financial burden borne by IVF patients. Moreover, the Human Reproduction centers involved in this study possess a oocyte donation program. Patients could donate their surpplus oocytes at any stage of their treatment, and those who agreed to join the program received a $50 \%$ discount in their overall treatment cost as an incentive. In the present study, 44 patients donated surplus oocytes before starting the treatment, while other 11 patients did so after concluding their treatment. However, donation was totally voluntary, and those couples who decided not to join the program had their wish respected, and any remaining surpplus oocytes were discarded at the end of their treatment.

In conclusion, this multicenter cohort prospective clinical study demonstrated that the vitrification of supernumerary oocytes may be an effective strategy in routine IVF programs, which could bring some important benefits to IVF patients.

Vitrification is fast becoming a reliable method to cryopreserve oocytes, permitting that more than one attempt at pregnancy may be performed with just one ovulation stimulation, reducing the financial burden borne by IVF patients. Warming and insemination of vitrified oocytes can be performed in synchrony with the endometrium window, which could lead to improved pregnancy rates. Increasingly, smaller number of embryos may have to be cryopreserved, reducing the possible religious, moral or legal problems associated to embryo storage. Finally, oocyte donation programs can help foster oocyte cryo-banks, benefitting a higher number of patients.

\section{Acknowledgements}

The authors would like to thank Mr. Antonio Carlos Correa for his support with the English version of the paper, the staff at Materbaby - Reprodução humana for their dedication to this study, and Ingámed (Maringá, Brazil) for kindly supplying the vitrification media used in this experiment.

\section{CONFLICT OF INTERESTS}

No conflict of interest have been declared.

\section{Corresponding author:}

Carlos Gilberto Almodin

Materbaby - Reprodução Humana e Genética

Maringá/PR, Brazil

E-mail: almodin@materbaby.com.br

\section{REFERENCES}

Al-Azemi M, Kyrou D, Kolibianakis EM, Humaidan $P$, Van Vaerenbergh I, Devroey P, Fatemi HM. Elevated progesterone during ovarian stimulation for IVF. Reprod Biomed Online 2012;24:381-8.

Al-Ghamdi A, Coskun S, Al-Hassan S, Al-Rejjal R, Awartani $\mathrm{K}$. The correlation between endometrial thickness and outcome of in vitro fertilization and embryo transfer (IVF-ET) outcome. Reprod Biol Endocrinol 2008;6:37.

Alikani M, Cohen J, Tomkin G, Garrisi GJ, Mack C, Scott RT. Human embryo fragmentation in vitro and its implications for pregnancy and implantation. Fertil Steril 1999;71: $836-42$.

Almodin CG, Minguetti-Câmara VC, Paixão CL, Pereira PC. Embryo development and gestation using fresh and vitrified oocytes. Hum Reprod 2010;25:1192-8.

Barnhart KT. Introduction: are we ready to eliminate the transfer of fresh embryos in in vitro fertilization? Fertil Steril 2014;102:1-2.

Borini A, Cattoli M, Bulletti C, Coticchio G. Clinical efficiency of oocyte and embryo cryopreservation. Ann N Y Acad Sci 2008;1127:49-58.

Bosch E, Labarta E, Crespo J, Simón C, Remohí J, Jenkins J, Pellicer A. Circulating progesterone levels and ongoing pregnancy rates in controlled ovarian stimulation cycles for in vitro fertilization: analysis of over 4000 cycles. Hum Reprod $2010 ; 25: 2092-100$.

Chang EM, Han JE, Kim YS, Lyu SW, Lee WS, Yoon TK. Use of the natural cycle and vitrification thawed blastocyst transfer results in better in-vitro fertilization outcomes: cycle regimens of vitrification thawed blastocyst transfer. J Assist Reprod Genet 2011;28: 369-74.

Chen QJ, Sun XX, Li L, Gao XH, Wu Y, Gemzell-Danielsson $\mathrm{K}$, Cheng LN. Effects of ovarian high response on implanta- 
tion and pregnancy outcome during controlled ovarian hyperstimulation (with GnRH agonist and rFSH). Acta Obstet Gynecol Scand 2007;86:849-54.

Cil AP, Seli E. Current trends and progress in clinical applications of oocyte cryopreservation. Curr Opin Obstet Gynecol 2013;25:247-54.

Cobo A, Kuwayama M, Perez S, Ruiz A, Pellicer A, Remohi J. Comparison of concomitant outcome achieved with fresh and cryopreserved donor oocytes vitrified by the Cryotop method. Fertil. Steril 2008;89:1657-64.

Cobo A, Meseguer M, Remohí J, Pellicer A. Use of cryo-banked oocytesin anoocytedonation programme: a prospective, randomized, controlled, clinical trial. Human Reprod 2010;25: 2239-46.

Cobo A, Diaz C. Clinical application of oocyte vitrification: a systematic review and meta-analysis of randomized controlled trial. Fertil Steril 2011;96:277-85.

Coutifaris C, Myers ER, Guzick DS, Diamond MP, Carson SA, Legro RS, McGovern PG, Schlaff WD, Carr BR, Steinkampf MP, Silva S, Vogel DL, Leppert PC; NICHD National Cooperative Reproductive Medicine Network. Histological dating of timed endometrial biopsy tissue is not related to fertility status. Fertil Steril 2004;82:1264-72.

Craft I, Forman R. What to do with spare embryos. Lancet $1996 ; 347: 1488-9$.

Kolibianakis EM, Venetis CA, Bontis J, Tarlatzis BC. Significantly lower pregnancy rates in the presence of progesterone elevation in patients treated with $\mathrm{GnRH}$ antagonists and gonadotrophins: a systematic review and meta-analysis. Curr Pharmaceut Biotechnol 2012;13: 464-70.

Kuwayama M, Vajta G, Kato O, Leibo SP. Highly efficient vitrification method for cryopreservation of human oocytes. Reprod Biomed Online 2005;11:300-8.

Kyrou D, Popovic-Todorovic B, Fatemi HM, Bourgain C, Haentjens P, Van Landuyt L, Devroey P. Does the estradiol level on the day of human chorionic gonadotrophin administration have an impact on pregnancy rates in patients treated with rec-FSH/GnRH antagonist? Hum Reprod 2009;24:2902-9.

Levron J, Yerushalmi GM, Brengauz M, Gat I, Katorza E. Comparison between two protocols for thawed embryo transfer: natural cycle versus exogenous hormone replacement. Gynecol Endocrinol 2014;30:494-7.

Loutradis D, Beretsos P, Arabatzi E, Anagnostou E, Drakakis $P$. The role of steroid hormones in ART. J Steroid Biochem Mol Biol 2008;112:1-4.

Pfeifer S, Goldberg J, McClure R, Lobo R, Thomas M, Widra E, Licht M, Collins J, Cedars M, Racowsky C, Vernon M, Davis O, Gracia C, Catherino W, Thornton K, Rebar R, La Barbera A. Mature oocyte cryopreservation: a guideline. Fertil Steril. 2013;99:37-43.

Potdar N, Gelbaya TA, Nardo LG. Oocyte vitrification in the 21st century and post-warming fertility outcomes: a systematic review and meta-analysis. Reprod Biomed Online. 2014;29:159-76.
Rienzi L, Romano S, Albricci L, Maggiulli R, Capalbo A, Baroni E, Colamaria S, Sapienza F, Ubaldi F. Embryo development of fresh 'versus' vitrified metaphase II oocytes after ICSI: a prospective randomized sibling-oocyte study. Hum Reprod 2010;25:66-73.

Robertson JA. Resolving disputes over frozen embryos. Hastings Cent Rep 1989;19:7-12.

Ruiz-Alonso M, Blesa D, Díaz-Gimeno P, Gómez E, Fernández-Sánchez M, Carranza F, Carrera J, Vilella F, Pellicer A, Simón $\mathrm{C}$. The endometrial receptivity array for diagnosis and personalized embryo transfer as a treatment for patients with repeated implantation failure. Fertil Steril 2013;100:818-24

Shapiro BS, Daneshmand ST, Garner FC, Aguirre M, Hudson C. Clinical rationale for cryopreservation of entire embryo cohorts in lieu of fresh transfer. Fertil Steril 2014; 102:3-9.

Siano L, Engmann L, Nulsen J, Benadiva C. A prospective pilot study comparing fertilization and embryo development between fresh and vitrified sibling oocytes. Conn Med 2013;77:211-7.

Trounson A, Mohr L. Human pregnancy following cryopreservation, thawing and transfer of an eight-cell embryo. Nature 1983;305:707-9.

Tucker MJ, Morton PC, Sweitzer CL, Wright G. Cryopreservation of human embryos and oocytes. Curr Opin Obstet Gynecol 1995;7:188-92.

Ubaldi F, Anniballo R, Romano S, Baroni E, Albricci L, Colamaria S, Capalbo A, Sapienza F, Vajta G, Rienzi L. Cumulative ongoing pregnancy rate achieved with oocyte vitrification and cleavage stage transfer without embryo selection in a standard infertility program. Hum Reprod 2010;25:1199-205.

Veleva Z, Karinen P, Tomás C, Tapanainen JS, Martikainen $H$. Elective single embryo transfer with cryopreservation improves the outcome and diminishes the costs of IVF/ ICSI. Hum Reprod 2009;24:1632-9.

Wang JG, Douglas NC, Dicken C, Nakhuda GS, Guarnaccia MM, Sauer MV. Cryopreservationof supernumerary high quality embryos predicts favorable outcomes for patients undergoing repeated cycles of in vitro fertilization. Fertil Steril 2008; 89:368-74.

Weineman R, Mainigi M. Why we should transfer frozen instead of fresh embryos: the translational rationale. Fertil Steril 2014;102:10-8.

Wong KM, Mastenbroek S, Repping S. Cryopreservation of human embryos and its contribution to in vitro fertilization success rates. Fertil Steril 2014;102:19-26.

Zhang SY, Lin XN, Song T, Tong XM, Shu J, Huang HF. [Gene expression profiles of peri-implantation endometrium in natural and superovulation cycles]. Natl Med J Chin (Chin) 2008;88:2343-6.

Zhou $F$, Lin $X$, Tong $X$, Li C, Liu L, Jin X, Zhu H, Zhang S. A frozen-thawed embryo transfer program improves the embryo utilization rate. Chin Med J 2009;122: 1974-8. 